\title{
EVALUACIÓN DEL RIESGO ERGONÓMICO DEL FARMACÉUTICO EN OFICINA DE FARMACIA CON EL MÉTODO REBA
}

\author{
ASSESSMENT OF THE ERGONOMIC RISK OF THE PHARMACIST IN THE PHARMACY \\ OFFICE WITH REBA METHOD
}

\section{Bernardo Prieto-Muñoz}

\begin{abstract}
Resumen: El estudio ergonómico busca identificar los riesgos más apremiantes de cada puesto de trabajo con el fin de realizar cambios necesarios que permitan mejorar la calidad de vida laboral de los trabajadores. Las lesiones debidas a la adopción de posturas forzadas provocan los llamados trastornos musculoesqueléticos que pueden dar lugar a incapacidad laboral. El objetivo de este trabajo fue valorar el grado de exposición del trabajador de farmacia al riesgo de adoptar posturas forzadas que generan fatiga y, a la larga, pueden provocar problemas de salud. Para estudiar las posturas forzadas del farmacéutico en la farmacia comunitaria se aplicó el método REBA, el cual se escogió por ser el más completo, ya que considera todas las partes del cuerpo. Tras realizar este estudio, se detectó que el farmacéutico adopta en su jornada laboral posturas perjudiciales que pueden dar lugar a trastornos musculoesqueléticos.
\end{abstract}

Palabras clave: Ergonomía, riesgos laborales, prevención de accidentes, postura, dolor musculoesquelético.

\begin{abstract}
The ergonomic study seeks to identify the most pressing risks of each job position in order to make the necessary changes to improve the quality of work life of workers. Injuries due to the adoption of forced postures cause so-called musculoskeletal disorders that can lead to incapacity for work. The objective of this work was to assess the degree of exposure of the pharmacy worker to the risk of adopting forced postures that generate fatigue and, in the long run, can cause health problems. To study the forced postures of the pharmacist in the community pharmacy, the REBA method was applied, which was chosen because it is the most complete, since it considers all parts of the body. After conducting this study, it was detected that the pharmacist adopts harmful postures during their working hours that can lead to musculoskeletal disorders.
\end{abstract}

Keywords: Ergonomics, occupational risks, accident prevention, posture, musculoskeletal pain.

Recepción: 15.09.2021 / Revisión: 08.11.2021 / Aceptación: 03.12.2021

\footnotetext{
"Departamento Farmacia y Tecnología Farmacéutica, Universidad Valencia. Valencia, España. Correo electrónico: bjprietom@gmail.com, priemu@alumni.uv.es. Orcid: https://orcid.org/oooo-0002-71748695
} 


\section{Introducción}

La ergonomía es el estudio sistemático de las personas en su entorno de trabajo con el fin de mejorar su situación laboral, sus condiciones de trabajo y las tareas que realizan. Su objetivo es adquirir información relevante y fiable que sirva de base para recomendar cambios en situaciones específicas y para desarrollar procedimientos que contribuyan a conseguir un entorno laboral seguro y satisfactorio. El estudio ergonómico de cada puesto de trabajo debe identificar las principales exigencias y los riesgos más apremiantes de los distintos puestos con el fin de realizar los cambios necesarios que permitan mejorar la calidad de vida laboral de los trabajadores (Laurig \& Vedder, 1998).

La primera referencia escrita de la palabra ergonomía data de 1857 en el libro "Compendio de Ergonomía o de la Ciencia del Trabajo basada en verdades de la naturaleza" del polaco Wojciech Jastrzebowki. Sin embargo, hay que esperar a 1949 a la fundación en Inglaterra de la primera sociedad de ergonomía: Ergonomics Research Society, para que el término ergonomía tenga el mismo significado que en la actualidad. Pero los problemas que interesan a la ergonomía son aún más antiguos, las lesiones relacionadas con el trabajo ya están descritas en el Antiguo Egipto y Leonardo da Vinci hizo numerosos estudios de la antropometría del ser humano y su relación con las máquinas, también investigó sobre los movimientos de los segmentos corporales, de tal manera que se le puede considerar como el precursor directo de la biomecánica moderna (Llorca-Rubio et al., 2015, p.23).

A finales del siglo XX las farmacias empezaron a cambiar, desde el lugar donde se dispensaban medicamentos con receta médica a lugares de diseño que incitaban al consumo de especialidades farmacéuticas publicitarias, cosméticos, gafas, bronceadores o productos de higiene. Las farmacias crecieron en metros cuadrados, escaparates, zonas diferenciadas con varios mostradores de colores, vitrinas y lugares donde la publicidad ocupaba un lugar destacado en contraposición con el aspecto sobrio y anticuado de la farmacia tradicional. El diseño ergonómico empezó a formar parte de la farmacia haciendo más apetecible y atractivo la estancia y la compra de productos sanitarios en las farmacias.

Esta evolución repercute necesariamente a la hora de evaluar los riesgos laborales y preparar su prevención. Hay que decir que la prevención de riesgos laborales en el entorno de trabajo no sólo es una exigencia legal, sino que constituye una demanda ética en cualquier organización, como puede ser una Farmacia Comunitaria.

El modelo activo de prevención, propuesto por el Instituto Sindical de Trabajo, Ambiente y Salud de Comisiones Obreras ([ISTAS-CCOO], 2017), obliga a ir por delante de los problemas. Así, las actuaciones más importantes de este modelo son:

- Planificar la prevención desde el momento mismo del diseño empresarial.

- Evaluar los riesgos actualizando la evaluación periódicamente cuando cambien las circunstancias.

- Adoptar un conjunto de acciones preventivas para eliminar y/o controlar los riesgos que se hayan detectado.

- Controlar la eficacia de las medidas preventivas adoptadas. 
- Integrar la acción preventiva a la empresa, en este caso es el farmacéutico titular el empresario.

- Informar a los trabajadores sobre los riesgos que comporta su trabajo.

- Formar a los trabajadores en materia preventiva.

- Establecer una vigilancia adecuada de la salud de los trabajadores.

- Desarrollar actuaciones ante situaciones de emergencia.

El objetivo principal de este trabajo es estudiar los posibles riesgos ergonómicos que padecen los trabajadores de una farmacia, utilizando para ello métodos basados en el estudio de la posición adoptada y el análisis de la carga física, según aconseja la Unión General de Trabajadores (UGT), como son la postura forzada, los movimientos repetitivos y el esfuerzo en la manipulación manual de cargas (UGT, 2017), y en el registro en el momento de la observación, mediante fotografías de los distintos segmentos articulares.

\section{Método REBA}

El método REBA (Rapid Entire Body Assessment) es un método de evaluación ergonómica propuesto por Sue Hignett y Lynn McAtamney, ergónomas e investigadoras de la ciudad de Nottingham. Fue publicado por la revista especializada, Applied Ergonomics, en el año 2000. $\mathrm{Su}$ objetivo es confeccionar un instrumento sensible que recogiera todo tipo de posturas de trabajo. El Instituto de Biomecánica de Valencia (IBV) lo describe como un método sencillo y útil destinado al análisis ergonómico de la carga postural. Basa sus resultados en la observación de diferentes posturas adoptadas por el trabajador durante el desarrollo de su función (IBV, 2021).

La descripción de las características más destacadas del método REBA, orientarán al evaluador sobre su idoneidad para el estudio de determinados puestos.

- Es un método especialmente sensible a los riesgos de tipo musculoesquelético.

- Divide el cuerpo en segmentos para ser codificados individualmente, y evalúa tanto los miembros superiores, como el tronco, el cuello y las piernas.

- Analiza la repercusión sobre la carga postural del manejo de cargas realizado con las manos o con otras partes del cuerpo.

- Considera relevante el tipo de agarre de la carga manejada, destacando que este no siempre puede realizarse mediante las manos, y por tanto, permite indicar la posibilidad de que se utilicen otras partes del cuerpo.

- Permite la valoración de la actividad muscular causada por posturas estáticas, dinámicas, o debidas a cambios bruscos o inesperados en la postura.

- El resultado determina el nivel de riesgo de padecer lesiones estableciendo el nivel de acción requerido y la urgencia de la intervención.

- Requiere de un equipamiento mínimo: lápiz y papel.

El método REBA evalúa el riesgo de posturas concretas de forma independiente. Por 
tanto, para evaluar un puesto se deben seleccionar, a priori, las posturas más representativas, que presentan mayor desviación respecto a la desviación neutra, y por ende, supongan una mayor carga postural. La selección correcta de las posturas a evaluar determinarán los resultados proporcionados por el método y las acciones futuras.

\section{Materiales y métodos}

\section{Fases del estudio ergonómico}

1. Observaciones previas a la evaluación: El técnico prevencionista definió todas las funciones que llevaba a cabo el farmacéutico y observó su entorno de trabajo, ya que es un estudio con un enfoque ergonómico. En este caso, el técnico identificó que el farmacéutico adoptaba posturas forzadas al subir al taburete para dispensar el medicamento solicitado.

2. Definición de objetivos: El técnico se fijó como objetivo principal modificar dentro de lo posible estas posturas forzadas.

3. Determinación de los factores y variables a estudiar: En este caso, el técnico estudió la altura del trabajador, de las cajoneras, del taburete, la frecuencia con que sube al último cajón, etc.

4. Selección de los métodos, técnicas e instrumentos a emplear: En esta ocasión se utilizó el método REBA.

Tabla 1. Puntuación de la actividad y nivel de acción método REBA.

\begin{tabular}{|c|c|c|c|}
\hline Nivel de acción & Puntuación & Nivel de riesgo & Intervención y posterior análisis \\
\hline 0 & 1 & Inapreciable & No necesario \\
\hline 1 & $2-3$ & Bajo & Puede ser necesario \\
\hline 2 & $4-7$ & Medio & Necesario \\
\hline 3 & $8-10$ & Alto & Necesario pronto \\
\hline 4 & $11-15$ & Muy alto & Actuación inmediata \\
\hline
\end{tabular}

Fuente. UPV (2021).

5. Establecimiento de criterios para la obtención de datos: Se observó al trabajador en su entorno, y después, previa su autorización, se realizaron fotografías y videos desde distintos puntos de toma.

6. Toma de datos: Se observaron detenidamente las fotografías, y se procedió a medir ángulos de brazos, piernas, flexión de cuello, etc.

7. Análisis de los datos y elaboración de conclusiones y recomendaciones: Esta es la parte más importante de la prevención de riesgos laborales, se debe llegar a conclusiones y recomendaciones para solucionar el problema detectado, si no es así, no tendría ninguna validez práctica el estudio. 


\section{Resultados y discusión}

Se ha elegido una farmacia representativa de la ciudad de Valencia, España. El estudio se llevó a cabo entre el lunes 5 de octubre y el viernes 29 de octubre de 2020, en jornada laboral completa. Se hizo un seguimiento de todos los movimientos y se tomaron numerosas fotografías cuando el trabajador adoptaba una postura que pudiera dar lugar a daño musculoesquelético. Al concluir el trabajo se llevó los datos numéricos a las tablas del método REBA elaboradas por la Universidad Politécnica de Valencia ([UPV], 2021) para obtener un valor numérico y obtener las conclusiones.

Se ha estudiado la postura que adopta el farmacéutico al dispensar una especialidad farmacéutica ubicada en la parte superior de una cajonera. Las cajoneras sustituyeron las antiguas estanterías como lugar donde se colocan los medicamentos; la ventaja es evidente por la capacidad de almacenamiento y el orden que consiguen. Las dimensiones de las cajoneras son las que el farmacéutico solicita al fabricante, suelen ser altas para tener mayor capacidad de almacenamiento, y aquí es donde radica el riesgo ergonómico, porque el trabajador se ve obligado a adoptar posturas forzadas, y en las que resulta incluso difícil mantener el equilibrio.

En la farmacia comunitaria estudiada el mueble estaba formado por tres cajoneras verticales unidas entre sí. La altura del conjunto llegaba a los 2,10 m, la anchura es de 1,30 m y la profundidad de $94 \mathrm{~cm}$. Cada cajonera está dividida en 16 cajones, cada uno de ellos de 12 cm de altura y $41 \mathrm{~cm}$ de anchura.

Figura 1. Estudio del ángulo del tronco, brazos, antebrazos y muñeca.

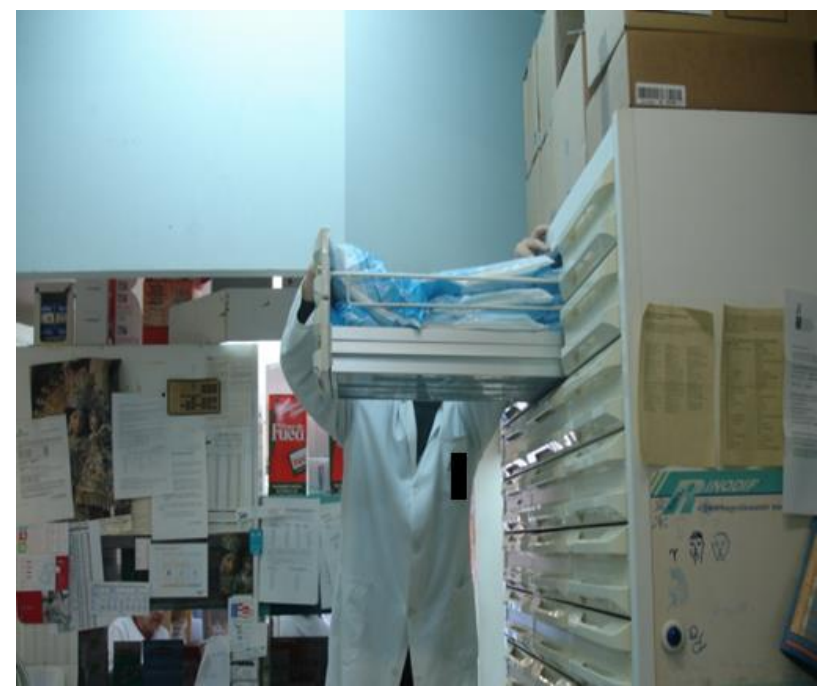


Figura 2. Estudio del ángulo del cuello, brazos, antebrazos y muñeca.

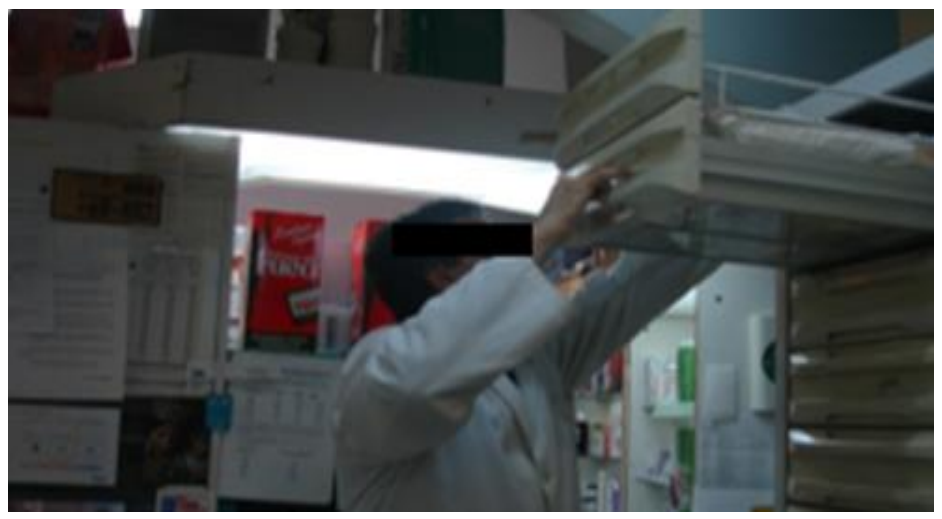

Figura 3. Estudio del ángulo de las piernas, brazos, antebrazos y muñecas.

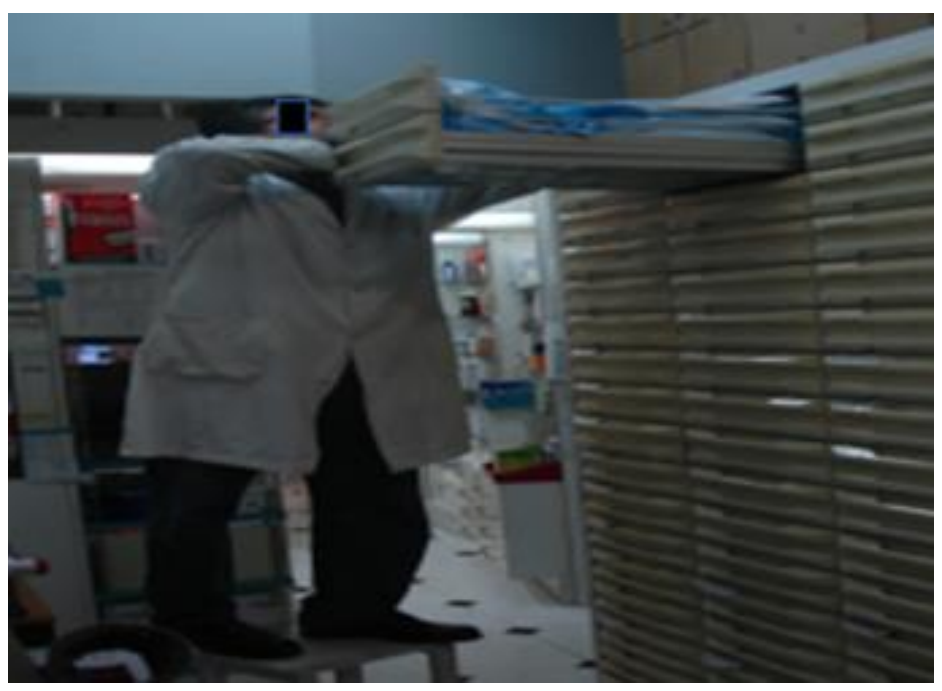

Figura 4. Estudio del ángulo del tronco, brazos y piernas.

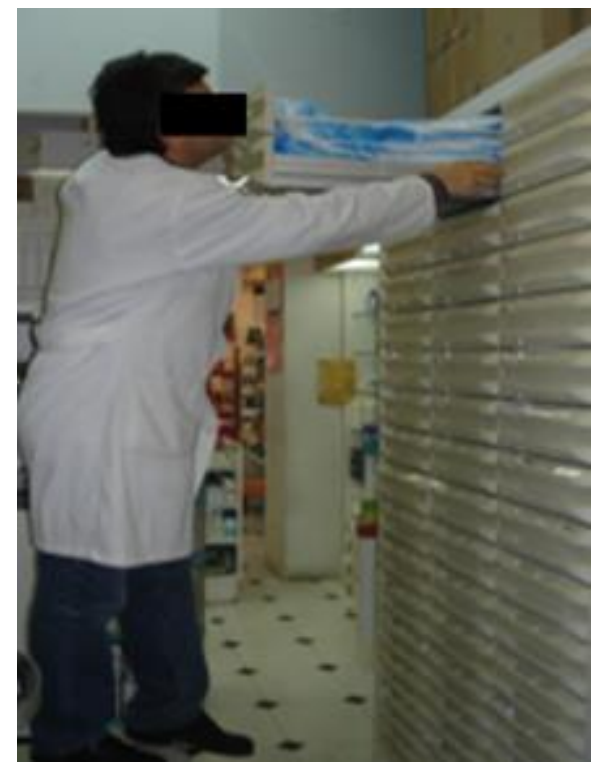


La información requerida por el método REBA de acuerdo con el Instituto Nacional de Seguridad y Salud en el Trabajo ([INSST], 2021) es básicamente la siguiente:

- Los ángulos formados por las diferentes partes del cuerpo (tronco, cuello, piernas, brazo, antebrazo, muñeca) con respecto a determinadas posiciones de referencia. Dichas mediciones pueden realizarse directamente sobre el trabajador (transportadores de ángulos, electrogoniómetros u otros dispositivos de medición angular), o bien a partir de fotografías, siempre que estas garanticen mediciones correctas (verdadera magnitud de los ángulos a medir y suficientes puntos de vista).

- La carga o fuerza manejada por el trabajador al adoptar la postura en estudio indicada en kilogramos.

- El tipo de agarre de la carga manejada manualmente o mediante otras partes del cuerpo.

- Las características de la actividad muscular desarrollada por el trabajador (estática, dinámica o sujeta a posibles cambios bruscos) (INSST, 2021).

Se procedió a la aplicación del método REBA, resumiendo las directrices de la UPV (2021) según los siguientes pasos:

- División del cuerpo en dos grupos, siendo el grupo A el correspondiente al tronco, el cuello y las piernas y el grupo B el formado por los miembros superiores (brazo, antebrazo y muñeca). Puntuación individual de los miembros de cada grupo a partir de sus correspondientes tablas.

- Consulta de la tabla para la obtención de la puntuación inicial del grupo A a partir de las puntuaciones individuales del tronco, cuello y piernas.

- Valoración del grupo B a partir de las puntuaciones del brazo, antebrazo y muñeca mediante la tabla correspondiente.

- Modificación de la puntuación asignada al grupo A (tronco, cuello y piernas) en función de la carga o fuerzas aplicadas, en adelante "puntuación A".

- Corrección de la puntuación asignada a la zona corporal de los miembros superiores (brazo, antebrazo y muñeca) o grupo B según el tipo de agarre de la carga manejada, en lo sucesivo "puntuación B".

- A partir de la "puntuación A" y la "puntuación B" y mediante la consulta de la tabla C se obtiene una nueva puntuación denominada "puntuación C".

- Modificación de la "puntuación C" según el tipo de actividad muscular desarrollada para la obtención de la puntuación final del método.

- Consulta del nivel de acción, riesgo y urgencia de la actuación correspondientes al valor final calculado.

La posición del tronco, cuello, piernas (grupo A), brazos, antebrazos y muñecas (grupo B) del farmacéutico se traslada a las tablas del método REBA. En rojo, la puntuación obtenida. Al estudiar las fotografías del trabajador se observa: 
- El tronco está flexionado en $20^{\circ}$, lo que corresponde a 2 puntos, y como está girado o inclinado a un lado, hay que sumar +1 . Puntuación: 3 .

- El cuello tiene una flexión entre $0^{\circ}$ y $20^{\circ}$. Puntuación: 1 .

- Se observa que hay apoyo unilateral del peso en una postura inestable. Puntuación de las piernas: 2.

- Los brazos tienen $45^{\circ}$ de extensión, lo que corresponde a 2 puntos, a los cuales se les suma +1 porque el hombro está levantado. Puntuación: 3 .

- Los antebrazos están extendidos. Puntuación: 2.

- Las muñecas están flexionadas entre $0^{0}$ y $15^{\circ}$. Puntuación: 1.

Sobre la tabla 4 se sitúa la puntuación del tronco, cuello y piernas, en el cruce de la columna con la fila correspondiente se indica el valor de la postura del tronco: 4. A esta puntuación se le suma el valor correspondiente a la carga o fuerza realizada en el momento de la dispensación de la especialidad farmacéutica, evidentemente pesa menos de $5 \mathrm{Kg}$. Hay que sumarle o, el código total A es de 4 .

La puntuación obtenida de las extremidades superiores, según la tabla 5, es de 4, a la que hay que sumar el dígito indicativo del tipo de agarre, en este caso el agarre es bueno, por tanto, hay que sumar o. La puntuación total es 4 .

Tabla 2. Estudio de tronco, cuello y piernas (grupo A).

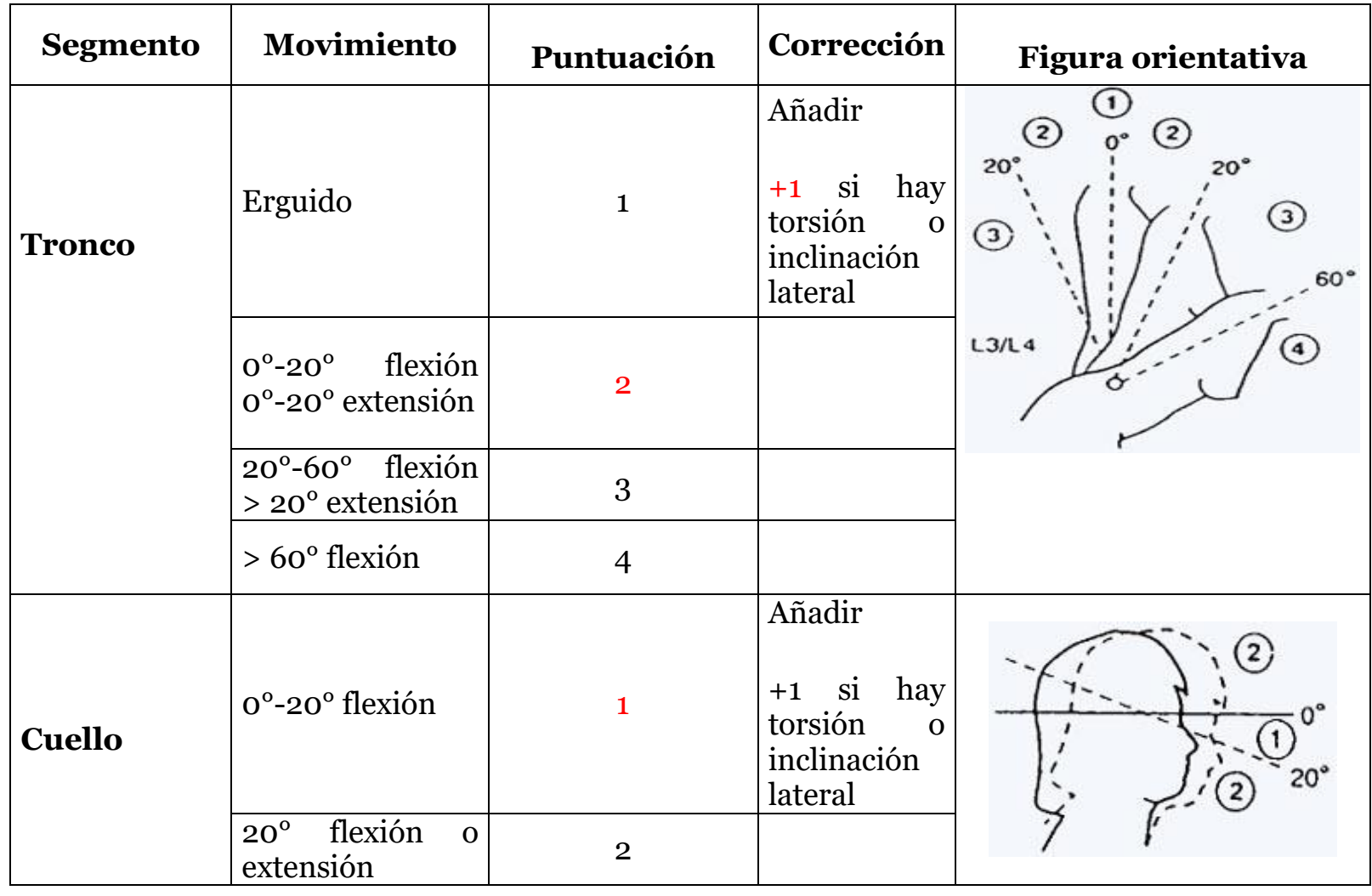




\begin{tabular}{|c|c|c|c|c|}
\hline \multirow[b]{2}{*}{ Piernas } & $\begin{array}{l}\text { Soporte } \\
\text { bilateral, } \\
\text { andando } \\
\text { sentado }\end{array}$ & 1 & $\begin{array}{l}\text { Añadir } \\
+1 \text { si hay } \\
+1 \text { dexión de } \\
\text { rodillas } \\
\text { entre } 30 \text { y } \\
60^{\circ}\end{array}$ & \multirow{2}{*}{$\left(\int^{(1)}\right.$} \\
\hline & $\begin{array}{l}\text { Soporte } \\
\text { unilateral, } \\
\text { soporte ligero o } \\
\text { postura inestable }\end{array}$ & 2 & $\begin{array}{l}+2 \text { si las } \\
\text { rodillas } \\
\text { están } \\
\text { flexionadas } \\
\text { más de } 60^{\circ} \\
\text { (salvo } \\
\text { postura } \\
\text { sedente) }\end{array}$ & \\
\hline
\end{tabular}

Fuente. UPV (2021).

Tabla 3. Estudio de brazos, antebrazos y muñecas (grupo B).

\begin{tabular}{|c|c|c|c|c|}
\hline \multirow[t]{4}{*}{ Brazos } & $\begin{array}{l}\text { o-20 } \\
\text { flexión/extensión }\end{array}$ & 1 & $\begin{array}{l}\text { Añadir } \\
+\quad 1 \text { si hay } \\
\begin{array}{l}\text { abducción } \\
\text { rotación }\end{array} \\
+\quad 1 \text { elevación } \\
\text { del hombro } \\
\text { - } 1 \text { si hay apoyo } \\
\text { o postura a } \\
\text { favor de la } \\
\text { gravedad }\end{array}$ & (2) \\
\hline & $>20^{\circ}$ extensión & 2 & & \\
\hline & $20-45^{\circ}$ flexión & 3 & & \\
\hline & $>90^{\circ}$ flexión & 4 & & \\
\hline \multirow{2}{*}{ Antebrazo } & $60^{\circ}-100^{\circ}$ flexión & 1 & & \\
\hline & $\begin{array}{l}<60^{\circ} \text { flexión } \\
>100^{\circ} \text { flexión }\end{array}$ & 2 & & (2) \\
\hline Muñecas & $\begin{array}{l}\mathrm{O}^{\circ}-15^{\circ}-\text { flexión/ } \\
\text { extensión }\end{array}$ & 1 & $\begin{array}{|lr|}\text { Añadir } & \\
& \\
+\quad 1 \text { si } & \text { hay } \\
\text { torsión } & 0 \\
\text { desviación } & \\
\text { lateral } & \\
\end{array}$ & $0=\ldots 0^{\circ}$ \\
\hline
\end{tabular}




\begin{tabular}{|l|l|l|l|l|}
\hline & $\begin{array}{l}>15^{\circ} \text { flexión/ } \\
\text { extensión }\end{array}$ & 2 & \\
\hline
\end{tabular}

Fuente. UPV (2021).

Tabla 4. Tabla puntuación grupo A.

\begin{tabular}{|c|c|c|c|c|c|c|c|c|c|c|c|c|c|}
\hline & & \multicolumn{12}{|c|}{ Cuello } \\
\hline & & & & & & & & & & & & 3 & \\
\hline \multicolumn{2}{|c|}{ Piernas } & $\mathbf{1}$ & 2 & 3 & 4 & $\mathbf{1}$ & 2 & 3 & 4 & $\mathbf{1}$ & 2 & 3 & 4 \\
\hline \multirow{5}{*}{ Tronco } & $\mathbf{1}$ & 1 & 2 & 3 & 4 & 1 & 2 & 3 & 4 & 3 & 3 & 5 & 6 \\
\hline & 2 & 2 & 3 & 4 & 5 & 3 & 4 & 5 & 6 & 4 & 5 & 6 & 7 \\
\hline & 3 & 2 & 4 & 5 & 6 & 4 & 5 & 6 & 7 & 5 & 6 & 7 & 8 \\
\hline & 4 & 3 & 5 & 6 & 7 & 5 & 6 & 7 & 8 & 6 & 7 & 8 & 9 \\
\hline & 5 & 4 & 6 & 7 & 8 & 6 & 7 & 8 & 9 & 7 & 8 & 9 & 9 \\
\hline \multicolumn{14}{|c|}{ Tabla carga/fuerza } \\
\hline \multicolumn{3}{|c|}{ o } & & \multicolumn{2}{|l|}{1} & \multicolumn{2}{|c|}{2} & \multicolumn{6}{|c|}{+1} \\
\hline \multicolumn{3}{|c|}{ Inferior a $5 \mathrm{~kg}$} & & \multicolumn{2}{|c|}{ 5-10 kg } & $10 \mathrm{k}$ & & \multicolumn{6}{|c|}{ Instauración rápida o brusca } \\
\hline
\end{tabular}

Fuente. UPV (2021).

Tabla 5. Tabla puntuación grupo B.

\begin{tabular}{|c|c|c|c|c|c|c|c|}
\hline & & \multicolumn{6}{|c|}{ Antebrazo } \\
\hline & & & $\mathbf{1}$ & & & 2 & \\
\hline \multicolumn{2}{|c|}{ Muñeca } & $\mathbf{1}$ & 2 & 3 & $\mathbf{1}$ & 2 & 3 \\
\hline \multirow{6}{*}{ Brazo } & $\mathbf{1}$ & 1 & 2 & 2 & 1 & 2 & 3 \\
\hline & 2 & 1 & 2 & 3 & 2 & 3 & 4 \\
\hline & 3 & 3 & 4 & 5 & 4 & 5 & 5 \\
\hline & 4 & 4 & 5 & 5 & 5 & 6 & 7 \\
\hline & 5 & 6 & 7 & 8 & 7 & 8 & 8 \\
\hline & 6 & 7 & 8 & 8 & 8 & 9 & 9 \\
\hline \multicolumn{8}{|c|}{ Agarre } \\
\hline \multicolumn{2}{|c|}{ o - Bueno } & \multicolumn{2}{|c|}{ 1- Regular } & \multicolumn{2}{|c|}{2 - Malo } & \multicolumn{2}{|c|}{3 -Inaceptable } \\
\hline
\end{tabular}




\begin{tabular}{|c|c|c|c|}
\hline $\begin{array}{c}\text { Buen agarre y fuerza } \\
\text { de agarre. }\end{array}$ & Agarre aceptable. & $\begin{array}{c}\text { Agarre posible pero no } \\
\text { aceptable }\end{array}$ & $\begin{array}{c}\text { Incómodo, sin } \\
\text { agarre manual. } \\
\text { Aceptable usando } \\
\text { otras partes del } \\
\text { cuerpo. }\end{array}$ \\
\hline Resultante grupo B + Resultante agarre. Puntuación B $=4$. \\
\hline
\end{tabular}

Fuente. UPV (2021).

Tabla 6. Puntuación total + actividad.

\begin{tabular}{|c|c|c|c|c|c|c|c|c|c|c|c|c|c|}
\hline \multirow{14}{*}{$\begin{array}{c}\text { Puntuación } \\
\text { A }\end{array}$} & \multicolumn{13}{|c|}{ Puntuación B } \\
\hline & & 1 & 2 & 3 & 4 & 5 & 6 & 7 & 8 & 9 & 10 & 11 & 12 \\
\hline & 1 & 1 & 1 & 1 & 2 & 3 & 3 & 4 & 5 & 6 & 7 & 7 & 7 \\
\hline & 2 & 1 & 2 & 2 & 3 & 4 & 4 & 5 & 6 & 6 & 7 & 7 & 8 \\
\hline & 3 & 2 & 3 & 3 & 3 & 4 & 5 & 6 & 7 & 7 & 8 & 8 & 8 \\
\hline & 4 & 3 & 4 & 4 & 4 & 5 & 6 & 7 & 8 & 8 & 9 & 9 & 9 \\
\hline & 5 & 4 & 4 & 4 & 5 & 6 & 7 & 8 & 8 & 9 & 9 & 9 & 9 \\
\hline & 6 & 6 & 6 & 6 & 7 & 8 & 8 & 9 & 9 & 10 & 10 & 10 & 10 \\
\hline & 7 & 7 & 7 & 7 & 8 & 9 & 9 & 9 & 10 & 10 & 11 & 11 & 11 \\
\hline & 8 & 8 & 8 & 8 & 9 & 10 & 10 & 10 & 10 & 10 & 11 & 11 & 11 \\
\hline & 9 & 9 & 9 & 9 & 10 & 10 & 10 & 11 & 11 & 11 & 12 & 12 & 12 \\
\hline & 10 & 10 & 10 & 10 & 11 & 11 & 11 & 11 & 12 & 12 & 12 & 12 & 12 \\
\hline & 11 & 11 & 11 & 11 & 11 & 12 & 12 & 12 & 12 & 12 & 12 & 12 & 12 \\
\hline & 12 & 12 & 12 & 12 & 12 & 12 & 12 & 12 & 12 & 12 & 12 & 12 & 12 \\
\hline & $+1: \mathrm{L}$ & na & as p & es d & cuer & est & cas, & $\mathrm{r}$ ej. & uan & as 1 & $\mathrm{se}$ & nin. & \\
\hline Actividad & $+1: 1$ & ovir & & pet & $\mathrm{OS}$, & r ej. & peti & $\mathrm{n} \mathrm{su}$ & rior & ve & $/ \mathrm{mi}$ & & \\
\hline & $+1: C$ & $\mathrm{amb}$ & po & ales & npol & ntes & pos & as i & tabl & & & & \\
\hline & & & ce & $\begin{array}{l}\mathrm{Pu} \\
\text { unt }\end{array}$ & $\begin{array}{l}\text { daci } \\
\text { ción }\end{array}$ & $\begin{array}{l}\text { tota } \\
\text { y pu }\end{array}$ & $\begin{array}{l}\text { el p } \\
\text { uaci }\end{array}$ & $\begin{array}{l}\text { sto } \\
\text { B) }\end{array}$ & ctiv & & & & \\
\hline & & & & & intu & ión & $\mathrm{al}=$ & & & & & & \\
\hline
\end{tabular}

Fuente. UPV (2021).

De acuerdo a la tabla 1, una puntuación REBA de 4, corresponde a un nivel de acción de 2, un nivel de riesgo medio, y por tanto, la intervención a realizar y posterior análisis es necesario.

\section{Conclusiones}

Al estudiar las posturas forzadas del farmacéutico con el método REBA, se concluye que existe un nivel de acción 2, un nivel de riesgo medio, y que la intervención y posterior análisis es necesario.

Las soluciones para mitigar estos riesgos no son difíciles, ya que en una oficina de farmacia los trabajos a realizar son numerosos y muy variados, el farmacéutico deberá rotar en sus funciones: atención al público en el mostrador, revisión de caducidades, realización y repaso de pedidos, gestión de las recetas, etc. En cuanto a la solución en el acceso a la parte superior de las cajoneras, se requieren medidas a adoptar por el método estudiado, tales como dejar inservible esta parte de la cajonera, o bien, almacenar en la parte superior medicamentos poco frecuentes o con mejor agarre, por ejemplo, jarabes, cuyas cajas 
normalmente son de mayor tamaño que la de los comprimidos. Un jarabe para la tos tiene una capacidad de $200 \mathrm{ml}$, es poco pesado y su agarre es bueno.

Para la prevención de las posturas forzadas se recomienda:

- Adecuado diseño de los planos de trabajo en cuanto a alturas y distancias de alcance para los brazos.

- Alternar, siempre que se pueda, trabajos de pie/sentado y sentado/andando.

- Dotar de silla ajustable para tareas que requieran estar sentado y silla-taburete para que el trabajador se pueda apoyar en tareas que requieran estar de pie.

- Establecer pausas para proporcionar el descanso muscular.

- Fortalecer la musculatura mediante el ejercicio físico.

- Formación en adopción de la postura correcta para realizar la tarea. 


\section{Referencias}

Instituto de Biomecánica de Valencia. (2021). Método REBA. http://www.ergoibv.com/blog/metodo-reba-evita-las-lesiones-posturales-2/

Instituto Nacional de Seguridad y Salud en el Trabajo. (2021). Herramientas prevención riesgos laborales para posturas de trabajo. https://www.insst.es/herramientas-de-prl-paraposturas-de-trabajo

Instituto Sindical de Trabajo, Ambiente y Salud de Comisiones Obreras. (2017). La prevención de riesgos laborales en los lugares de trabajo. Guía para una intervención sindical. https://ib.ccoo.es/33e92egb5d7ceode41b4990565dc3911000061.pdf

Laurig, W., \& Vedder, J. (1998). Ergonomía. Organización Internacional del Trabajo. https://www.insst.es/documents/94886/161958/Cap\%C3\%ADtulo+29.+Ergonom\%C3\%AD $\underline{\mathrm{a}}$

Llorca-Rubio, J. L., Llorca-Pellicer, L., \& Llorca-Pellicer, M. (2015). Manual de ergonomía aplicada a la prevención de riesgos laborales. Ediciones Pirámide. https://www.misgsst.com/public/documento/AyuW8byo53.pdf

Unión General de Trabajadores. (2017). Ergonomía. Riesgos ergonómicos. UGT-Madrid Ediciones.

https://madrid.ugt.org/sites/madrid.ugt.org/files/manual riesgos ergonomicos 2019 on line def $0 . p d f$

Universidad Politécnica de Valencia. (2021). Método REBA. Evaluación de posturas forzadas. https://www.ergonautas.upv.es/metodos/reba/reba-ayuda.php 